\title{
Social Characters for Computer Games
}

\author{
Bill Tomlinson \\ ACE (Arts Computation Engineering) program, University of California, Irvine \\ 430A Computer Science Building, Irvine, CA 92612 USA \\ Email:wmt@uci.edu
}

\begin{abstract}
Many current video games feature virtual worlds inhabited by autonomous 3D animated characters. These characters often fall short in their ability to participate in social interactions with each other or with people. Increasing the social capabilities of game characters could increase the potential of games as a platform for social learning. This article presents advances in the area of social autonomous character design. Specifically, a computational model of social relationship formation is described. This model formed the basis for a game entitled "AlphaWolf" that allows people to play the role of newborn pups in a pack of virtual wolves, helping the pups to find their place in the social order of the pack. This article offers the results from a 32-subject user study that assessed the social relationship model, showing that it effectively represents the core elements of social relationships in a way that is perceivable by people. Additionally, this article proposes a game that will allow parents, teachers and children to experiment with computational social behavior through social virtual characters. This research contributes to the development of games for social learning by offering a set of viable algorithms for computational characters to form social relationships, and describing a project that could utilize this model to enable children to learn social skills by interacting with game characters.
\end{abstract}

Keywords: Computer Games, Video Games, Autonomous Characters, Social Dominance

\section{Introduction}

Most modern video games feature interactive 3D animated characters. At the time of writing, nine of the ten top-selling PC games in the US, and eight of the ten top-selling console games in the US, feature 3D animated virtual worlds inhabited by real-time autonomous characters (Gamasutra.com, 2004b, Gamasutra.com, 2004a). (The other three games in these top twenty also feature characters, but in 2D worlds.) The characters in these games have many strengths. They are delicately modeled, carefully animated and elaborately rendered, all at real-time frame rates. Despite these capabilities, game characters on the whole lack the complex social competence to participate in the relationships that form the heart of human interactions.

Video games show great potential as platforms for social learning (Jenkins, 2002). If games are to serve as an effective platform for social learning, the characters in those games could benefit from the ability to engage in social relationships with each other and with people. This article presents a robust computational model that enables game characters to form social relationships. It offers an original evaluation of this computational model that supports its validity. In addition, it proposes a game, based on the computational model, through which children, parents and teachers 
may explore social interactions by interacting with social autonomous characters.

This research was developed as part of a project called AlphaWolf, an interactive game developed at the MIT Media Lab. This game featured a pack of six virtual wolves - three fully autonomous adults and three semiautonomous pups whom people could direct by howling, growling, whining or barking into microphones. The focus of the game was the social relationships that the wolves formed with each other, enabled by a novel computational model of emotional memories.

Figure 1 shows an example of the animated images that a player saw in AlphaWolf. The graphical buttons at the bottom of the screen show the player which other wolves their pup has met. The posture of the wolf on each button shows the mental picture that the player's wolf has of that other wolf. For example, a wolf whom the player's wolf knows to be submissive to it would be shown rolled over on its back in a typical wolf submission posture.

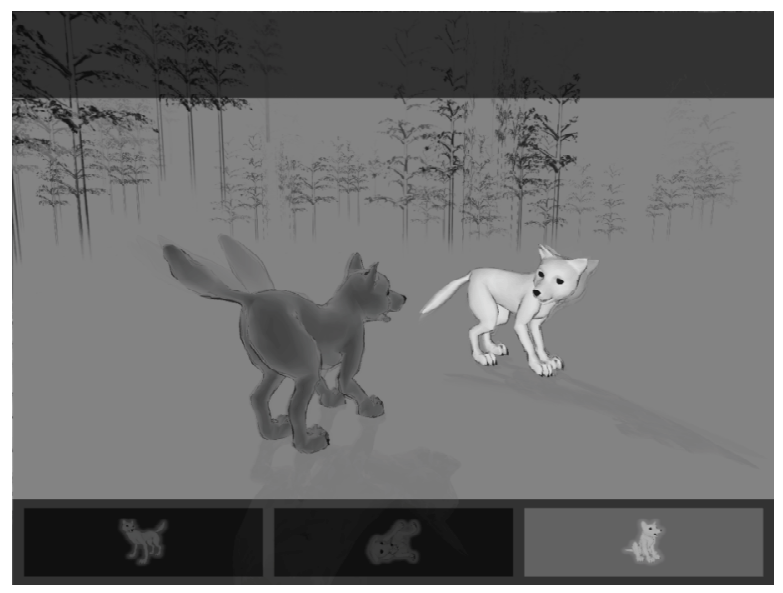

Figure 1: Two virtual wolf pups from the AlphaWolf game.

The evaluation of this model was conducted by means of a 32-subject human user study. Results from this evaluation support the hypothesis that the relationship model enables computational systems to form social relationships in a way that is perceptible by people.

A direction for future work that was inspired by AlphaWolf is a social learning game that allows children, parents and teachers to explore social relationships together by interacting with a community of autonomous characters. This game is described in more detail below.

Socially capable autonomous characters are an important building block for character-based games in general (Tomlinson and Blumberg, 2002), and are particularly relevant to games that focus on social learning. This article presents recent advances in computational modeling of social relationships, including a quantitative evaluation of a working model. In addition, it proposes a system for incorporating this model into a game for learning about social skills. This work contributes to the basic research that will facilitate further explorations of socially capable autonomous characters and the social learning games in which they will be situated.

\section{Related Work}

The development of computational systems with social competence is an active area of research in a number of fields, including autonomous agents and multi-agents systems (Hales and Edmonds, 2003), human computer interaction (Bickmore and Picard, 2004), computer graphics (Tomlinson and Blumberg, 2002), robotics (Vaughan et al., 2000), artificial intelligence (Dautenhahn, 2000), video games (Mateas and Stern, 2003) and artificial life (Breazeal et al., 2004). This section describes the work in these and other areas that are most relevant to the research presented here.

\section{Autonomous Characters}

The computer/video game industry has expended great effort to make compelling realtime characters. Many of these games showcase cutting edge research in graphics, character motion, AI and other areas. Games such as EA's The Sims have focused on game characters, building characters with simple personalities and interaction dynamics. Nevertheless, most game characters are weak in areas such as expressiveness and believability.

Academic researchers have looked more closely at the topics of expressiveness and believability, focusing most frequently on interactive virtual humans (e.g., (Gratch et al., 2002)). Perlin and his colleagues have done pioneering work in creating synthetic actors (e.g., (Perlin and Goldberg, 1996)). By applying 
procedural modifiers to characters' motion and offering an engine for scripting the characters' behaviors, they have created virtual characters who move and interact very naturally. Blumberg and his colleagues have contributed in several areas including action-selection (Isla et al., 2001), learning (Blumberg et al., 2002) and interactive cinematography (Tomlinson et al., 2000). Cassell and her colleagues built virtual humanoids with the ability to express themselves like real people, in particular in the area of embodied conversational agents (e.g., (Bickmore, 2003, Cassell et al., 1999)). Bates and his colleagues have created computational characters who appear lifelike and are able to interact with people in real time (Bates et al., 1992, Reilly, 1996). More recently, Schaub and his colleagues have presented research on empathic characters (Schaub et al., 2003). Prendinger and his colleagues offered an evaluation mechanism for empathic characters based on skin conductivity and other physiological signals (Prendinger et al., 2004). Eladhari and Lindley offer a design for a virtual mind for player characters (Eladhari and Lindley, 2003). Lee and colleagues have looked at ways of controlling the motions of avatars (Lee et al., 2002). Robots have also been endowed with characteristics that allow them to engage people in expressive ways (Velasquez, 1998, Breazeal, 2000). Many other researchers have also contributed to the creation of compelling synthetic characters, e.g., (Cavazza et al., 2002, Funge et al., 1999, Paiva et al., 2001, Thalmann et al., 1997, Hodgins and Pollard, 1997).

Despite the abundance of research in virtual characters, the field is still in its infancy. As one pair of researchers put it, "[t]he ethos of the tireless, constant, friendly human companion can quickly become an ethos of a broken, very nonhuman machine." (Randall and Pedersen, 1998, p. 68).

\section{Social Behavior}

Throughout this research, a social relationship will be defined as "a learned and remembered construct by which an entity keeps track of its interaction history with another entity, and allows that history to affect its current and future interactions with that entity" (Tomlinson, 2002, p. 22-23). Social relationships serve as mechanisms for context preservation (Cohen et al., 1999), allowing an entity to behave differently toward different social partners, rather than interacting with all other entities in the same way. Social relationships are closely linked with emotion, both in their formation (Damasio, 1994) and in their expression (Darwin, 1965 (originally published 1872)). Damasio offers the Somatic Marker Hypothesis, which proposes that people (and animals) attach emotional significance to stimuli that they encounter in their environment, and then reexperience those emotions when they encounter those stimuli on future occasions.

Various models of emotion have been proposed. Ekman (1992), for example, offered a categorical model with six cardinal emotions in humans - happiness, sadness, anger, fear, surprise and disgust. Another model of emotion maps a range of emotional phenomena onto an explicitly dimensioned emotional space; Mehrabian and Russell (Mehrabian and Russell, 1974) offer an emotion space defined by pleasure, arousal and dominance. Panksepp draws a distinction between basic emotions, such as anger and fear, and social emotions, such as love and grief (Panksepp, 1998). Nardi offered three dimensions of connection that contribute to social relationships - affinity, commitment, and attention (Nardi, 2005); these dimensions will be included in the evaluations of human relationships with autonomous characters. Social relationships also form the building blocks from which many social networks may be formed (Watts, 2003, Fisher and Dourish, 2004, boyd, 2004).

\section{Computational Model}

The social relationship mechanism described and evaluated in this paper combines Damasio's Somatic Marker Hypothesis (Damasio, 1994) with Mehrabian and Russell's dimensional model of emotions (Mehrabian and Russell, 1974) to create social relationships in a pack of virtual wolves. These relationships are mechanisms by which context is preserved among different dyadic pairs of wolves (Cohen et al., 1999). The behavior of the virtual wolves is informed by the study of real wolves (e.g., (Mech et al., 1998, Fox, 1971, Klinghammer and 
Goodmann, 1985)), and by previous work in synthetic social systems (e.g., (Hemelrijk, 1996)). Wolves, humans and other primate species all exhibit social hierarchies of various forms (Mech, 1999, Wrangham et al., 1994, US_Army, 2004), so it is possible that some of the relationship structures developed for wolves may have some application to humans as well. Immerman and Mackey (2003) point to possible convergent evolution of social behavior between humans and canids.

The social relationship mechanism is situated in the Synthetic Characters Group's Toolkit, described in (Burke et al., 2001), and (Isla et al., 2001). In the evaluation section, the mechanism is compared to other mechanisms by which entities may interact, in particular those described in (Vaughan et al., 2000). In addition, the AlphaWolf installation is evaluated with similar criteria to those used by other believable social and emotional agent systems - e.g., (Reeves and Nass, 1996, Reilly, 1996, Perlin and Goldberg, 1996, Cassell et al., 1999, Breazeal, 2000). Inspired by Craig Reynolds' Boids (Reynolds, 1987), we have tried to keep the mechanism as simple as possible. None of the systems that we have encountered uses an emotional memory mechanism to enable longterm social relationships among computational entities.

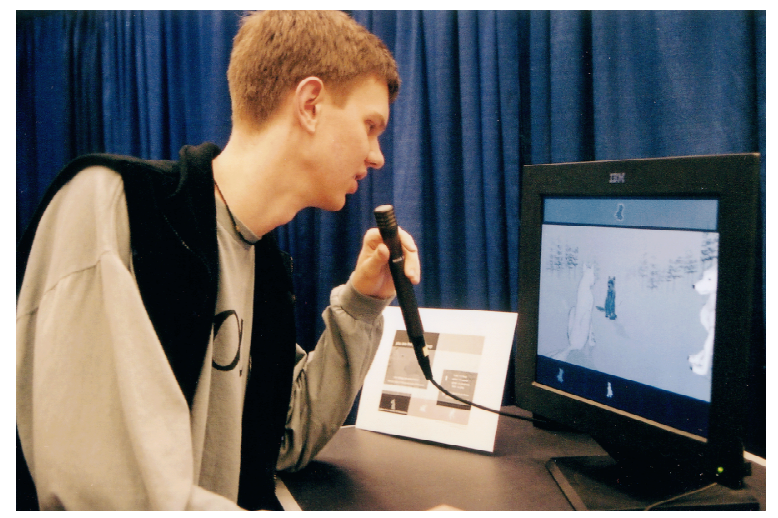

Figure 2: A participant interacts with the wolves using a microphone interface.

\section{The AlphaWolf Game}

The computational model of social relationship formation described in this article was originally designed as part of an interactive game entitled AlphaWolf. In the game, three participants help direct the actions of virtual wolf pups in a simulated litter. By howling, growling, whining or barking into a microphone, each participant can tell her pup to howl, dominate, submit or play (see Fig. 2). The actions that the pup takes affect emotional relationships that the pup forms with its littermates and with the adults of the pack. The pups autonomously maintain these relationships and display them by means of the emotional style in which they take the actions suggested by the participants. The relationships are also displayed to the participant through dynamic buttons at the top and bottom of each screen, which show each of the social partners of that wolf in a dominant or submissive pose that reflects how the participant's pup views that partner.

There are six wolves in all, three pups and three adults. The wolves take various colors black, white or gray. Each puppy grows up from pup to adult size over approximately five minutes. By the end of the five-minute interaction, the pups, guided by the users, have worked out their places in the social order of the pack.

AlphaWolf premiered in the Emerging Technologies section of SIGGRAPH 2001 (Tomlinson et al., 2001), and has been exhibited in seven venues in five countries since that time. To see a short video describing the project, please view a short segment from 10/22/02 edition of the Public Broadcasting Service (PBS) television show "Scientific American Frontiers" with host Alan Alda, available at:

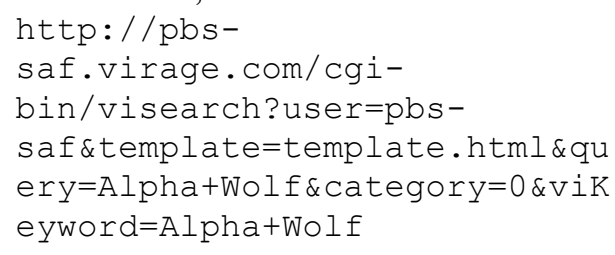

\section{Computational Model of Social Relationships}

The mechanism by which the virtual wolves form social relationships with each other involves models of emotion, perception and learning. The relationships in turn are expressed through the behavior of the wolves. This section elaborates on each of these elements. 


\section{Emotion}

The model of emotion in AlphaWolf is very simple - a single floating-point value called dominance, which varies from 0.0 to 1.0 . (Dominance is a subset of a more complex dimensional representation of emotion presented by Mehrabian and Russell (Mehrabian and Russell, 1974) including pleasure and arousal as two other orthogonal axes). Each wolf's dominance value is affected by its interactions. For example, being bitten causes a wolf's dominance to drop, and being the target of another wolf's submission causes a wolf's dominance to increase.

The wolf's emotional state affects the style in which the wolf takes its actions. For example, a wolf with a current dominance value near 0.0 might walk with its tail between its legs and its ears back, while a wolf with a higher dominance value will hold its tail and ears erect. The system blends between example animations to give an expressive range to the behavior of the wolves (Downie, 2001).

In addition, when a wolf is behaving autonomously, its emotional state affects what actions it chooses to take towards its social partners. For example, a submissive wolf might choose to roll over on its back, while a dominant wolf might growl.

\section{Perception}

The second major section of the relationship system is the way in which the wolves perceive each other. The wolves are able to tell their pack mates apart in order to form different relationships with each of them. They also know when they are in an interaction with each other. (For example, being bitten is a much different experience than seeing someone else get bitten.)

The wolves disambiguate among their social partners by means of a unique identification tag. Being able to identify social partners is one of the three principles that make up the "bedrock of cooperation" (the other two being that individuals must be likely to encounter each other more than once, and that individuals must have information about how the individual has behaved in the past - see below) (Kollock, 1998, Axelrod, 1984). While it may seem simplistic for entities to "know" each other by means of a unique ID, real wolves appear to be able to distinguish each other by scent (Beaver, 1999).

From the point of view of an individual wolf $\mathbf{A}$, an interaction with a partner $\mathbf{B}$ begins when $\mathbf{A}$ has $\mathbf{B}$ as its Object of Attention, and perceives that $\mathbf{B}$ is reciprocally attending to him. For $\mathbf{A}$, the interaction ends when it changes its Object of Attention so that it is no longer attending to $\mathbf{B}$, regardless of whether $\mathbf{B}$ is still attending to $\mathbf{A}$. In fact, $\mathbf{A}$ would have no way of telling whether $\mathbf{B}$ was still attending to it, since that information should be unavailable to it unless $\mathbf{B}$ is its Object of Attention. Giving an individual only one Object of Attention does preclude the social relationship mechanism from making correct associations in certain circumstances (e.g., attending to one wolf in front, while being bitten by another wolf from behind). However, this simplified form should be able to be extended to involve more complex perceptual mechanisms. In addition, if a wolf bit me from behind every time I attended to a certain social partner, I would not be surprised if that affected my relationship with that partner, rather than just with the wolf doing the biting.

To summarize, a character is able to identify individuals, to have an Object of Attention, and to assess when it is the Object of Attention of another individual.

\section{Learning}

The first time individual $\mathbf{A}$ ends an interaction with individual $\mathbf{B}$, it forms an "emotional memory" of $\mathbf{B}$. The model of an emotional memory contains three pieces of information the unique ID of $\mathbf{B}$, an emotional value, and a confidence value. ${ }^{1}$ When the emotional memory is first formed, the emotional value stores the emotion that $\mathbf{A}$ was feeling at the time its interaction with $\mathbf{B}$ ended. Since the interactions that $\mathbf{A}$ has had with $\mathbf{B}$ may have altered its emotional state over the course of their interaction, forming the emotional memory at the end of the interaction rather than at the beginning will reflect the emotional content of the relationship more accurately.

The next time $\mathbf{A}$ switches to have $\mathbf{B}$ as its Object of Attention, its emotional memory of $\mathbf{B}$ will influence its current emotional state in proportion to its confidence in that model. The 
emotional memory is applied to the current emotional state by:

$$
E^{\prime}=\left(C \times E_{m}\right)+((1-C) \times E)
$$

Equation 1

where $\mathrm{E}^{\prime}$ is the wolf's new emotional value, $\mathrm{C}$ is the confidence value of the emotional memory being applied, E is the wolf's emotional value prior to the application of the emotional memory, and $\mathrm{E}_{\mathrm{m}}$ is the emotional value that is stored in the emotional memory.

At the end of each successive interaction, $\mathbf{A}$ revises its emotional memory of $\mathbf{B}$. Upon revision, two of the three elements of an emotional memory are changed - confidence and emotional value. Confidence is revised before the emotional value so that the change in the emotional value will reflect the change in confidence, thereby preserving the effect of deviations from the expected emotional interaction.

The confidence value is revised by:

$$
\mathbf{C}^{\prime}=\mathrm{C}+\left((\operatorname{Min}(\mathrm{C}, 1-\mathrm{C})) \times\left(\mathbf{T}_{\mathrm{c}}-\left|\mathbf{E}_{\mathrm{m}}-\mathbf{E}\right|\right) \times \mathbf{L}\right)
$$

\section{Equation 2}

where $C^{\prime}$ is the new confidence value for the emotional memory, $\mathrm{C}$ is the previous confidence, $T_{c}$ is some confidence threshold between 0 and $1, \mathrm{E}_{\mathrm{m}}$ is the emotional value that is stored in the emotional memory, $\mathrm{E}$ is the wolf's current emotional state, and $\mathrm{L}$ is a learning rate. (Multiplying the learned component by the Min of $\mathrm{C}$ and 1-C effectively clamps the confidence value to between 0 and 1 , and helps to polarize relationships.)

The emotional value stored in the emotional memory is then revised by:

$$
\mathbf{E}_{\mathbf{m}}{ }^{\prime}=\left(C \times \mathbf{E}_{\mathbf{m}}\right)+((\mathbf{1}-\mathbf{C}) \times \mathbf{E})
$$

\section{Equation 3}

where $E_{m}{ }^{\prime}$ is the new emotional value stored in the wolf's emotional memory, $\mathrm{C}$ is confidence,
$E_{m}$ is the emotional value in the memory prior to the revision, and $\mathrm{E}$ is the wolf's current emotional state.

These equations represent a simple but effective implementation of the emotional memory mechanism. For more elaborate social behavior, any or all of these equations might be made more complex.

The emotional memories described here function as remembered constructs by which an individual keeps track of its interaction history with another individual. They allow that history to affect its current and future interactions with that individual. These two elements satisfy the definition of a social relationship offered earlier.

\section{Expression}

Each wolf's emotional state affects what it does and how it does it. The most sublimely complex representation of a social relationship isn't of much use without an equally expressive range of behavior. The AlphaWolf system uses a motor blending system described in (Downie, 2001) to enable the characters have dynamic expressive ranges (see Figure 3). Downie's expressive motor system allows a wolf's current emotional state to affect the style in which the wolf behaves (e.g., run dominantly or submissively). Since emotional memories affect the wolf's current emotional state, they also affect the style of the wolf's behavior.

\section{Evaluation}

To evaluate the effectiveness of the social relationship mechanism in AlphaWolf, a set of user studies was conducted. Several hypotheses were tested through these experiments, of which the one presented here was most central. For a discussion of the other hypotheses and their results, please see (Tomlinson, 2002). This section presents the core hypothesis, experimental method, results and discussion in

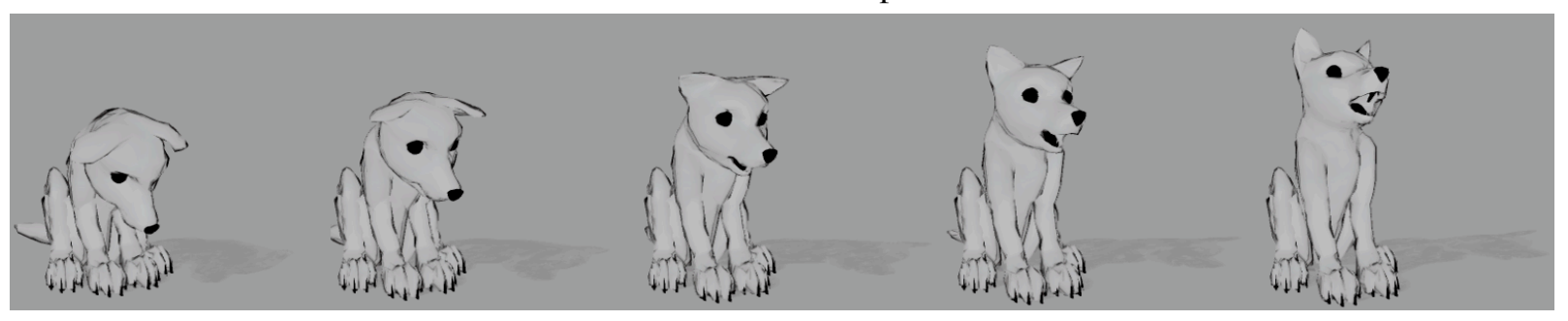

Figure 3: An emotional range from submissive to dominant. The extreme poses were hand-crafted by an animator. The center three poses are automatically generated. 


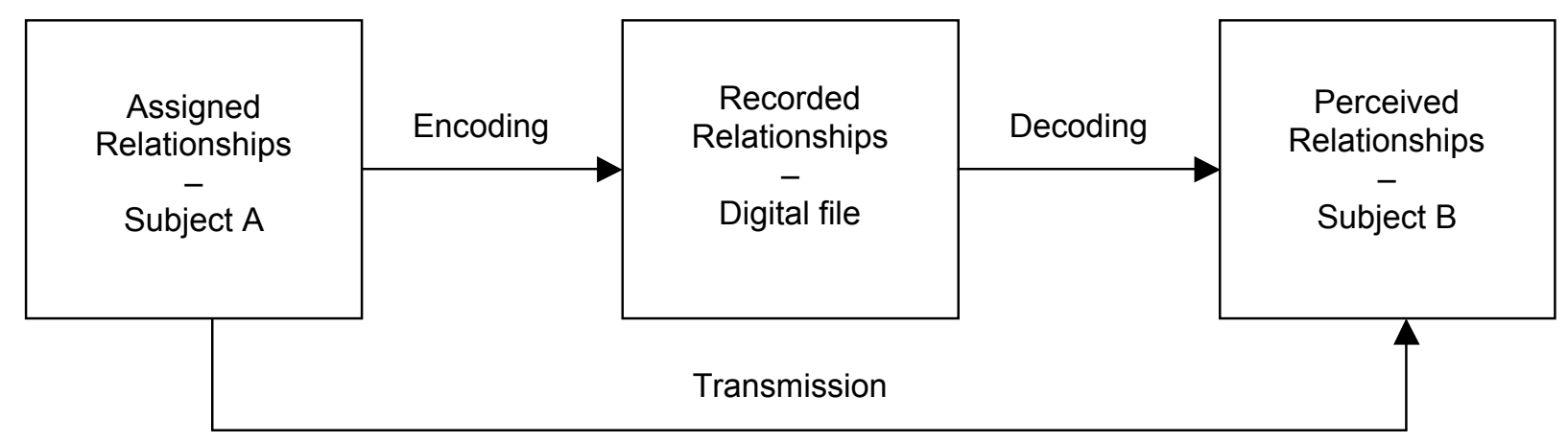

Figure 4: Shannon's model of communication, adapted to represent the transmission of social information between two subjects in the AlphaWolf study.

this study.

\section{Hypothesis}

The central hypothesis of this study was as follows: the mechanism in AlphaWolf encodes social relationships created by a first person in a way that can be perceived by a second, naïve person. This hypothesis has two subhypotheses: a) the mechanism successfully encodes social relationships, and b) naïve users can effectively perceive the relationships among the characters.

\section{Experimental Method}

This hypothesis was evaluated by means of an experiment involving 32 human subjects. The subjects ranged in age from 17 to 55 (mean $=$ 26.2, standard deviation $=7.8$ ). Half $(16)$ were female $(\min$ age $=18, \max$ age $=55$, average age $=26.1$, standard deviation $=10.1$, and half were male $(\min$ age $=17$, $\max$ age $=37$, average age $=26.4$, standard deviation $=4.7$ ).

In the user tests, each of the 32 subjects interacted with the AlphaWolf game. Each subject participated in three 4-minute interactions with different versions of the game. Two of each subject's runs were interactive, and a third run was non-interactive.

In the interactive runs, all subjects directed the gray pup, and were instructed to form specific social relationships with its siblings, the white and black pups. Various runs featured the AlphaWolf mechanism (described earlier in this paper) or one of three alternate relationship mechanisms (Random, Emotional or Fixed), which lacked the persistent, expressive qualities of the AlphaWolf mechanism. At the end of each interactive run, the relationships of the pups were written to a file.

The non-interactive runs for each subject featured pups with relationships that were loaded in from the file created by a previous subject's interaction. Subjects simply sat and watched the wolves interact, and observed the relationships that they exhibited.

The two interactive and one non-interactive runs occurred in random order.

The evaluation of the hypotheses above draws upon Shannon's Theory of Communication (Shannon, 1948) (see Figure 4). Shannon's theory subdivides the process of communication into five parts - an information source, a transmitter, a channel, a receiver and a destination. Each of these parts has a corresponding element in the AlphaWolf user tests. Each act of communication involved two subjects - Subject A and Subject B. Subject A's session with the wolves preceded that of Subject B; one of Subject A's runs created the relationships that were loaded into Subject B's Non-interactive run.

The simple model presented by Shannon offered a clear way to represent an act of social communication. Subject A is assigned the task of forming two social relationships. He or she encodes these relationships into a computational representation through an interactive run of the AlphaWolf system. These relationships are loaded in at the beginning of Subject B's Noninteractive run. If Subject B can perceive the relationships, they have been successfully decoded. If both encoding and decoding work, transmission occurs. This model allowed the 
experiment to determine which elements of the system were capturing or transmitting the social information effectively.

\section{Information Source}

The first stage in the communication of social relationships is specifying the relationship to be transmitted. These relationships were specified in advance - the experimenter informed Subject A of the relationships that he or she should attempt to have the gray pup form with the white pup and the black pup. All subjects were instructed to be dominant to one pup and submissive to the other. Therefore, the experimenter is the information source, and the assigned relationships are the message that might or might not be transmitted to the destination.

\section{Transmitter}

Subject A's interaction with the virtual wolf pack is the transmitter, taking the assigned relationships and converting them into the AlphaWolf dominance/confidence representation. This conversion encodes the relationships in a way that can be transmitted over the channel.

\section{Channel}

Whereas Shannon's theory deals primarily with a noisy channel (a telephone cable, for example), the channel in the experiment was a digital file that was loaded in without any loss of precision (barring experimenter error). This file stored the relationships formed by Subject A's interaction until they were loaded into Subject B's Noninteractive run.

\section{Receiver}

Subject B's interaction serves as the receiver, decoding the social relationship representation into a human-readable form. (While the current representation is simple enough that it, too, is somewhat human-readable, a more elaborate version with three emotional axes, for example, would be completely opaque.) By viewing the social interactions among the virtual wolves in a Non-interactive run, Subject B attempts to recognize the relationships among the pups.

\section{Destination}

The final destination of the message is the questionnaire that Subject B fills out. If Subject $\mathrm{B}$ reliably perceives the same relationships that were assigned to Subject A, and the only link between the two subjects is the AlphaWolf representation, then it is very likely that the representation successfully transmitted the relationships.

The dissection of the process of communication in the above sections and in Figure 4 demonstrates that there might be difficulty at a variety of points in a single act of communication. In the experiment, Subject A might not understand the instructions. He or she might fail to encode the relationships because of a failure of the interface. There could be problems writing the file at the end of Subject A's run, or loading it at the beginning of Subject B's. Subject B could fail to recognize the relationships. Subject B could get confused and fill out the form incorrectly. The experimental method was constructed in order to minimize the chance of each of the peripheral problems and to focus on the two central issues at hand - whether Subject A can encode the social relationships, and whether Subject B can decode them. Only if both of these conditions are true can the AlphaWolf system be said to transmit information that people would attribute to a social relationship.

Strong graphics and animation are key components of this system. Muddy graphics or poor animation could certainly render the system non-functional, preventing people from recognizing the relationships of the wolves. However, the results do not rely solely on the graphics. Without the underlying relationship representation, Subject B might accurately perceive certain relationships, but these relationships could have no correlation with those assigned to Subject A. Graphics and animation are crucial to the transparency of the system, but do not in themselves account for the relationship mechanism's functioning.

\section{Results}

This section describes the results of the encoding, decoding and transmission elements of the above study.

\section{Encoding}

Each of the 32 subjects viewed one Noninteractive run during their session. This Noninteractive run was randomly assigned to be the first second or third run that the subject 
encountered. Thirty-one of the 32 subjects viewed Non-interactive runs based on other subjects' interactive runs. (The first subject had a Non-interactive run based on an interactive run from the pilot study, which had a slightly different setup. That subject will therefore not be included in certain portions of this analysis.)

Half of these runs were encoded by previous subjects interacting with runs that featured the AlphaWolf mechanism, while the other half were encoded by the Random algorithm or the Fixed algorithm. Of those encoded with the AlphaWolf mechanism, half again were done through the Emotional algorithm in which emotional memories are still formed, even though they are not allowed to influence a wolf's current emotional state. Each run included two assigned relationships, one between the gray pup and the white pup, and one between the gray pup and the black pup.

To determine whether or not encoding had occurred, the assigned relationship was compared to the recorded representation of the relationship. The relationship between two wolves ( $\mathbf{A}$ and $\mathbf{B})$ is determined by an average of the dominance values, weighted by the confidence values:

$$
\frac{\left(\left(D_{A B} \times C_{A B}\right)+\left(\left(1-D_{B A}\right) \times C_{B A}\right)\right)}{\left(C_{A B}+\mathbf{C B A}_{B}\right)}
$$

\section{Equation 4}

where $\mathrm{D}_{\mathrm{AB}}$ is $\mathbf{A}$ 's dominance value with respect to $\mathbf{B}, \mathrm{C}_{\mathrm{AB}}$ is $\mathbf{A}$ 's confidence in that dominance, $D_{B A}$ is $\mathbf{B}$ 's dominance value with respect to $\mathbf{A}$, and $\mathrm{C}_{\mathrm{BA}}$ is $\mathbf{A}^{\prime}$ 's confidence in that dominance.

This formula yielded a single value from 0.0

to 1.0 that captured the essence of the relationship between the two wolves. If this value was less than 0.5 , the relationship was "submissive" from the point of view of the gray pup. If it was greater than 0.5 , the relationship was "dominant." Because of the relative complexity of the system, it was exceedingly unlikely that a relationship would ever equal exactly 0.5 . However, if two pups never met (as sometimes occurred between the black and the white pups, who might accidentally pass the four minute run without ever meeting), the value would end up at exactly 0.5 (a weighted average of the two starting values, 0.5 and 0.5 ). This condition did not happen in any of the assigned relationships involving the gray pup, though, and is therefore not relevant to these results.

Of the 32 total relationships encoded with the AlphaWolf mechanism (16 runs $x 2$ relationships per run), the internal representation matched the assigned relationship in 30 cases, and did not match in 2 cases $(93.8 \%$ success, $p$ $<0.0001^{2}$, see Figure 5). These figures demonstrate that the subjects and the system were quite successful at encoding social relationships.

Even in subjects' first run of virtual wolves, 15 out of 16 were encoded successfully $(93.8 \%$ success, $\mathrm{p}=0.0021$ ). These figures demonstrate that there was not a significant learning curve in the experiment; people were just as successful at the beginning of the session as they were at the end.

In the runs without the AlphaWolf mechanism, in which relationships were either predetermined or random, 20 of the 30 runs were encoded correctly $(66.7 \%$ success, $\mathrm{p}=0.1261)$. While pure randomness would give $50 \%$ encoding, the $\mathrm{p}$ value demonstrates that it is reasonable that these data arose by chance. A larger sample size would be necessary to determine for certain if the elevated encoding resulted from some code error or problem in the experimental method, or simply from five wrong rolls of the dice.

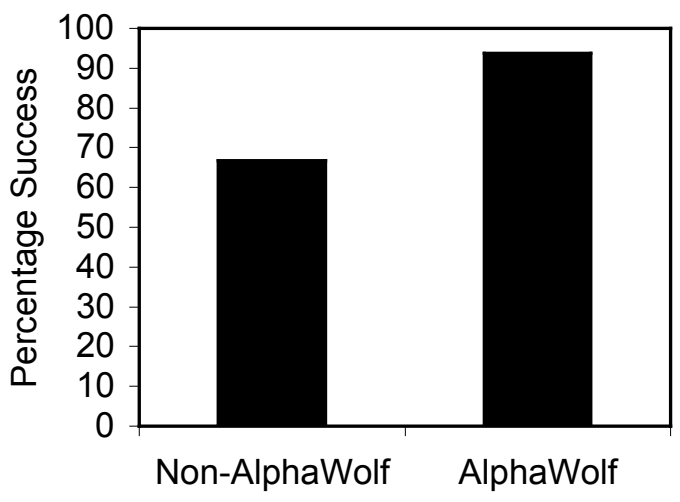

Figure 5: Encoding success with Non-AlphaWolf and AlphaWolf social relationship mechanisms. The AlphaWolf mechanism offers significantly improved encoding over random chance, or over alternate mechanisms.

One 29-year-old male subject wrote a comment that suggests a possible reason the 
encoding success rate wasn't even higher. He reported a "[s]trong urge to test relationships growl at black, for instance." The fact that people like to "poke at" a system is an important consideration in the design of interactive systems. Because the AlphaWolf system allows the computational entities to switch their relationships in response to adequate user input (e.g., it took about three clear interactions, in which both pups took on opposite roles, to reverse a relationship) the urge for people to vary their behavior could have had a significant impact on the results. Nevertheless, it appears that encoding was largely successful despite the possibility of intentional disobedience.

\section{Decoding}

There were 31 subjects whose Non-interactive runs featured relationships created during another subject's run. Each of these Noninteractive runs included two relationships involving the gray pup (gray-black and graywhite). In the questionnaire at the end of that run, subjects were asked to specify which of each pair of pups was more dominant.

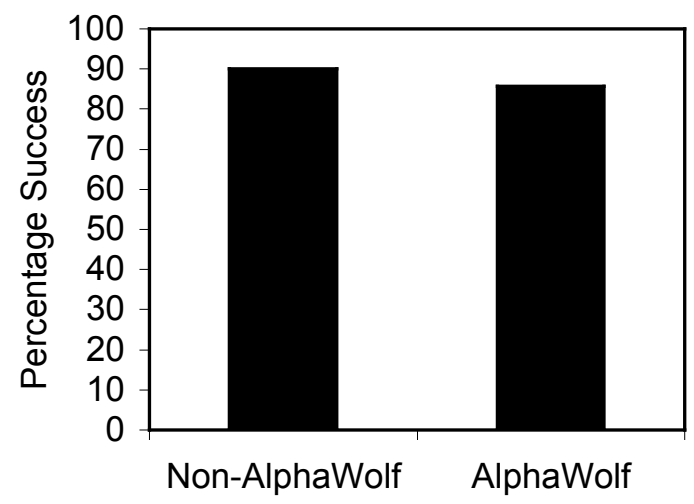

Figure 6: Decoding success with Non-AlphaWolf and AlphaWolf social relationship mechanisms. Decoding is successful regardless of relationship mechanism.

The way in which a match in decoding was determined was to simplify both the recorded representation and the subjects' answers to the questionnaire. Using Equation 4 above, the representation was reduced from four numbers to one, which in turn was converted to a simple "dominant" or "submissive" by the method described above. So, for example, if Wolf A had a dominance of 0.7 and a confidence of 0.5 with respect to Wolf $\mathrm{B}$, and Wolf $\mathrm{B}$ had a dominance of 0.1 and a confidence of 0.8 with respect to wolf $\mathrm{A}$, the equation would reduce to:

$$
\begin{aligned}
& ((0.7 \times 0.5)+((1-0.1) \times 0.8)) /(0.5+0.8)= \\
& (0.35+0.72) / 1.3= \\
& 0.82
\end{aligned}
$$

Since 0.82 is greater than 0.5 , Wolf A would be considered to be dominant with respect to Wolf B.

Subjects' responses to the questionnaires were also simplified, with an answer of 1,2 or 3 counting as "submissive", an answer of 5, 6 or 7 counting as "dominant", and an answer of 4 counting as "couldn't tell."

Of the 62 relationships (31 runs $\times 2$ relationships per run), subjects were able to determine which pup was dominant in 53 cases, couldn't tell in 2 cases $^{3}$, and chose incorrectly in 7 cases $(88.3 \%$ success, $p<0.0001$, see Figure $6)$. These figures, well above chance levels, show that people were able to watch a run of virtual wolves and comprehend the relationships that they had seen there. Subjects were successful at decoding social relationships, according to the definition offered earlier: "a learned and remembered construct by which an entity keeps track of its interaction history with another entity, and allows that history to affect its current and future interactions with that entity" (Tomlinson, 2002, p. 22-23)).

Even in their first run when they could not have learned about which animations correlate with dominance and submission from previous interactive runs, subjects were successful at "reading" the relationships among the pups; of 22 first run relationships, subjects decoded 18 correctly, couldn't tell for 1 , and chose incorrectly in $3(85.7 \%$ success, $\mathrm{p}=0.0048)$. These figures demonstrate that fully naïve subjects were able to determine relationships from only their pre-existing knowledge and their viewing of real wolves; they did not just learn what this system "meant" by social relationships over the course of their interactive runs.

There was no significant difference between decoding success when the relationships had been encoded by the AlphaWolf mechanism versus when they had been encoded by a random or fixed mechanism. Of the 32 decoded from AlphaWolf-created relationships, 26 were 
correct, 2 couldn't tell, and 4 were incorrect (86.7\% success, $\mathrm{p}<0.0001)$. Of the 30 decoded from non-AlphaWolf origins, 27 were correct and 3 were incorrect $(90.0 \%$ success, p < 0.0001). This result suggests that having the AlphaWolf encoding mechanism in the first stage of the transmission did not significantly disrupt the decoding process in the second stage.

The Relationships between Black and White

Although the black and white pups were forming relationships with each other as well as with the gray pup, these pups were not the camera system's "lead actor," and therefore it was not very common for the camera to capture a clear dominance interaction between the two. As one 30-year-old male subject put it: "It was hard to determine the relationship between the two other pups (in this case between white \& black) as I rarely saw them interacting with each other (only at the end)." Added to which, only the two relationships involving the gray wolf were assigned to the encoding subjects. Therefore, the relationships between black and white were not included in the above results for decoding.

Nevertheless, it appears that subjects did just as well decoding the relationships between these two pups as they did in the relationships involving the gray pup. Of the 32 black/white relationships, subjects successfully decoded 26, subjects couldn't tell in 2 cases, got it wrong in 2 , and in 2 cases the black and white pups never met $^{4}$ (and therefore had no relationship) (92.9\% success, $\mathrm{p}<0.0001)$. Even in their first run, subjects were successful; of the 11 subjects whose Non-interactive run was in the first position, there were 10 correct decodings, 1 run where the pups did not form relationships, and no mistakes. ( $100 \%$ success, $\mathrm{p}=0.0079) \mathrm{It}$ appears that it doesn't take much screen-time for people to recognize a relationship.

\section{Transmission}

Considering the entire process (both encoding and decoding) as a single act of communication, there were 16 subjects whose Non-interactive runs were based on another subject's interactive run that featured the AlphaWolf algorithm. Each of these subjects' Non-interactive runs had two relationships, for a total of 32 relationships. Of these, 26 were successfully transmitted (assigned relationship matched recorded representation and answer given on questionnaire), 1 was a double error resulting in successful communication of the message (assigned relationship matched answer given on questionnaire, but recorded relationship was opposite $\left.{ }^{5}\right), 2$ couldn't tell, and 3 were incorrect (89.7\% success, $\mathrm{p}<0.0001$, see Figure 7 ). These figures verify that the AlphaWolf algorithm and the entire AlphaWolf system successfully captures, transmits and displays social relationships in a way that is readily apparent to humans. The fact that subjects were able to decode the wolves' behaviors successfully regardless of the encoding mechanism suggests that the animation system was working in a way that was apparent to people. The most important feature of the AlphaWolf relationship algorithm is that it enabled people to encode the wolves' relationships in a format that could later be perceived by a separate subject.

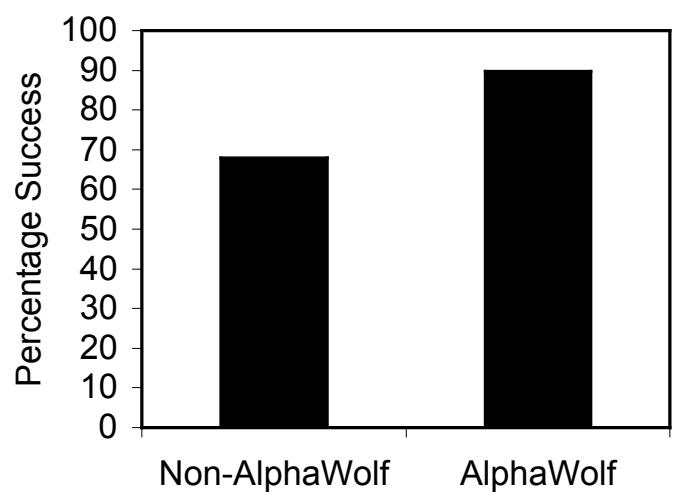

Figure 7: Transmission of relationships with NonAlphaWolf and AlphaWolf social relationships. Transmission is significantly better with the AlphaWolf mechanism than with the alternate mechanisms or by random chance.

Transmission was much less successful in cases where the relationships had not been encoded by the AlphaWolf mechanism. Of the 30 cases where encoding occurred by one of the other three mechanisms, transmission occurred in 19 cases, there were 2 double errors, and 9 incorrect responses $(67.9 \%$ success, $\mathrm{p}=0.0574)$. These figures are a bit high (in line with the slightly elevated encoding described above), but well below the level attained by the AlphaWolf mechanism. The two double errors are appear to be partially responsible for the artificially high 
number. The disparity between the transmission in the AlphaWolf and Non-AlphaWolf cases suggests that the success of the AlphaWolf cases was due to the AlphaWolf algorithm (rather than any other cause), since all other factors were held constant.

\section{Summary and Discussion}

To summarize the above study: 32 human subjects interacted with packs of virtual wolves under controlled conditions. Each person played the role of a gray wolf pup and attempting to direct that pup to form certain dominance relationships with its two siblings. At the end of the run (approx. 4 minutes long), the system recorded all of the pups' internal representation of the relationships. A second experimental subject later viewed a Non-interactive pack of autonomous wolves whose relationships were specified by the recorded relationships from the previous subject's interactive run. If the second subject's perception of the relationships matched the relationships that had been assigned to the first subject, then the AlphaWolf system succeeded in encoding, transmitting and decoding those relationships. This method of analysis is derived from Shannon's Theory of Communication (Shannon, 1948).

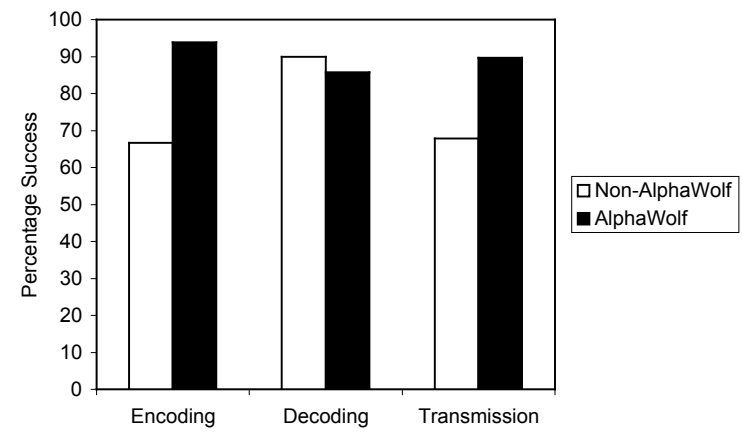

Figure 8: The AlphaWolf mechanism offers significantly better encoding of relationships than chance $(50 \%)$ or than a system without the AlphaWolf mechanism. Since there were no statistically significant differences in decoding, the greater success at encoding translated directly to significantly improved transmission.

The results from that study demonstrate that the AlphaWolf mechanism successfully represents social relationships (see Figure 8). Using the AlphaWolf mechanism, the first subjects successfully encoded $93.8 \%$ of the relationships that they had been assigned to create $(\mathrm{p}<0.0001)$. The second subjects decoded $86.7 \%$ of the relationships that they viewed $(p<0.0001)$. These two factors resulted in a successful transmission rate of $89.7 \%$ ( $<<$ 0.0001). Each of these figures demonstrates significantly better performance than the $50 \%$ success rate predicted by chance.

Using the non-AlphaWolf mechanism, $66.7 \%$ of the encoded relationships matched those that had been assigned to the first subject $(\mathrm{p}<0.1261)$. The second subjects decoded $90.0 \%$ of the relationships that they viewed $(\mathrm{p}<$ $0.0001)$. These two factors resulted in a successful transmission rate of $67.9 \%(\mathrm{p}<0$. 0574). Both encoding and transmission are not statistically different from chance (although, as discussed above, they are somewhat higher than the predicted value of $50 \%$ ).

The significantly lower levels of encoding and transmission among non-AlphaWolf runs confirm that these effects are a direct result of the AlphaWolf algorithm. The human user evaluation of the AlphaWolf system supports the hypothesis that the system and its relationship algorithm successfully encode, decode and transmit a valid representation of social relationships.

Nevertheless, this system is still greatly simplified from wolf social relationships, let alone from human relationships. It could be argued that the system doesn't scale well $89.7 \%$ accuracy on a single-axis model of emotion drops down to $72.2 \%$ accuracy on a three-axis model. However, having an exact prediction of emotion is not absolutely necessary in a model such as this, and in fact might not reflect the realities of biological relationships. Confusion over emotions is standard in human relationships, and a system that is very good, but not perfect, might more accurately model the natural phenomenon. Also, the partial successes of the model (where, for example, it would predict two of the three axes effectively) would still provide reasonable approximations of emotionally responsive behavior. Therefore, this model should scale reasonably well, and in biologically plausible ways. Despite the simplification of the system to represent only one emotional axis in this case, it should provide 
a solid grounding for more complex relationship systems for modeling both animals and humans.

It could also be argued that creatures that always exhibit the emotions that they are feeling do not capture the complexities of human social and emotional interactions. This is a fair point, and this system is limited in that it does not allow entities to mask their emotional responses as humans sometimes do. However, in order to mask one's emotions, it is necessary for one to have emotions first. This system does not capture the full complexity of human (or even wolf) relationships, but it does provide a central building block that could be used to develop more believable emotions and relationships.

\section{Limitations of the Study}

There are a number of limitations to the design of this study. First, it is possible that the "social relationships" transmitted by the system are not real social relationships, but instead just a way of transmitting patterns of behaviors that humans associate with simple social relationship phenomena. This difference could be relevant on two levels. First, there is a practical difference, where perhaps a more complex or effective computational mechanism could do a better job of capturing social relationships than the AlphaWolf mechanism. If this is the case, then hopefully AlphaWolf can provide a starting point or inspiration for the design of this superior mechanism. A deeper issue is whether a computational simulation can have a social relationship, or whether social relationships are by definition the domain of human beings. Similar questions have been discussed in various other aspects of computational systems (e.g., intelligence, emotion). The author prefers a functional definition of social relationships that would allow computational systems to form them, but respects that other people would disagree with this position.

A second limitation is that the AlphaWolf mechanism captures only simple dyadic relationships, and does not explicitly represent relationships among three or more individuals. Since people are able to comprehend triadic relationships, subjects may have used this skill to intuit more information about the wolves' relationships than the experimenters expected. For example, if a decoding subject had a strong view of the gray pup's relationship with the black pup and a strong view of gray's relationship with white, and if the encoding subjects were always assigned to play gray, to dominate one pup, and to submit to the other, then, by the transitive property of social relationships, subjects might be able to predict the relationship between black and white. Nevertheless, packs encoded with the random algorithm would arguably detract from this phenomenon, and pups encoded during a Fixed run would have strong relationships, but would not be guaranteed to have gray in the middle rank. Despite these facts, it is relevant that certain "whole-world" effects may have contributed to people achieving some indirect understanding of relationships to which they were not well-exposed directly.

A third limitation of this study involves the relatively small sample size used in it. While 32 subjects were adequate to find statistical significance in several important areas, there were additional findings that might have been more effectively understood with more additional data, such as the effectiveness of various interaction paradigms (e.g., microphone vs. keyboard) at enabling subjects to encode relationships.

\section{Proposed Interactive Game for Examining Social Behavior}

During the development of AlphaWolf, it became apparent that subtle changes to the social relationship algorithms could have sweeping repercussions on the personalities of the characters. Different relationship algorithms might lead to "Might makes right" characters, "Do unto others as you would have them do unto you" characters, "An eye for an eye" characters, or pacifist characters. In addition, changing the perceptual mechanism so that the characters recognized each other by color rather than by unique ID led to characters that exhibited simple social stereotyping, or even racist, behavior.

The design of systems like AlphaWolf is a subtle and potentially problematic domain, since they deal with human-like behaviors and ultimately will have an effect on the humans who interact with them. It is very easy for implicit biases to find their way into the design 
of the system. By thinking through the potential social ramifications of the system, the designers of AlphaWolf attempted to be aware of the ideologies that were embedded in the system's code. Raybourn addressed similar issues in describing the design of intercultural agents (Raybourn, 2003).

The realization of the profound impacts of small modifications to the relationship code has led to a potentially interesting direction for future work - creating a game that allows people to explore the ramifications of these kinds of social interactions. This game will be designed so that children, teachers and parents are able to learn about social behavior together. This system will be based on the core research described above. The system will feature a virtual environment with a community of interactive animated characters in it. People will be able to control several relevant algorithms in the characters' social relationship mechanisms. This control will allow them to explore and discuss the ramifications of various kinds of social behavior. While the social phenomena will be vastly simplified compared to the complexities of human social behavior, they will nevertheless provide scaffolding through which parents, teachers and children may talk about these issues and hopefully come to understand them better. In addition, by introducing students to the interactions between computer programming and interactive animation, the system could encourage students to consider pursuing programming and computer science in general as careers.

Prior to the deployment of the system, several schools and families will be sought out to serve as a user group for evaluating the effectiveness of the system. The system will be evaluated in two ways. First, the parents and teachers in the user group will be asked to complete questionnaires before and after using the system to assess whether they found it effective. Second, the children involved will be asked to interact with a version of the system before and after interacting with it with their parents/teachers, and will fill out questionnaires regarding why they made certain decisions in social situations in the game. Their responses will be analyzed to see if they gave more considered responses in the post-case. After the initial evaluation and revision, the system will be released on the web so that families and schools are able to download and utilize it.

\section{Conclusion}

Entertainment-oriented video games are largely based in virtual worlds inhabited by avatars and autonomous characters. In order for learning games to compete with these games, they too may need to have compelling animated characters. Giving these characters social competence will make it much more likely that they could provide opportunities for social learning to the people who interact with them. This article has offered a simple computational model for enabling autonomous characters to have social capabilities. In addition, it provided quantitative support for the effectiveness of the model, by means of a 32-subject user study. Finally, it described a potential game in which these social characters could be situated to provide an opportunity for people to explore a range of social phenomena through interactions with virtual characters.

This research clearly only scratches the surface of social algorithms for autonomous characters. Nevertheless, it points toward a fruitful area of research into the creation of computational characters as building blocks for educational games. Social characters could serve as virtual teachers, synthetic students and learning companions, and could help provide rich virtual worlds in which people may learn and grow.

\section{Acknowledgements}

The author would like to thank several anonymous reviewers who contributed significantly to its improvement of this paper. This research would not have been possible without the collaboration and support of the other members of Professor Bruce Blumberg's Synthetic Characters Group at the MIT Media Lab, in particular Marc Downie, Matt Berlin, Jesse Gray, Adolph Wong, Derek Lyons, Jennie Cochran, Bryan Yong and Michael Patrick Johnson. The research also benefited greatly from discussions with Professors Bruce Blumberg, Cynthia Breazeal, Rosalind Picard and Richard Wrangham. 


\section{Notes}

${ }^{1}$ Although the social relationship mechanism that we describe treats individuals as emotional significant stimuli, a stimulus does not have to be an individual - only a causative entity (Damasio, 1994). Forming emotional memories of other kinds of stimuli (e.g., the presence of two wolves at the same time) could result in other kinds of relationships (e.g., alliance formation).

2 Throughout this analysis, the Mann-Whitney U test was used to calculate the $\mathrm{p}$ values.

${ }^{3}$ For the purposes of calculating averages and $p$ values, runs in which subjects couldn't tell the relationship were discarded.

${ }^{4}$ For the purposes of calculating averages and $p$ values, runs in which pups never formed a relationship were discarded.

${ }^{5}$ Throughout this analysis, double errors were thrown out in determining percentages and $\mathrm{p}$ values. Nevertheless, it is an interesting possibility that "whole-world" effects somehow contribute to the prevalence of double errors - that some aspect of the interrelation among the three pups makes people able to divine the correct relationship despite a flawed representation. In this particular double error, the recorded relationships suggested that both pups had decided that they were submissive to the other. This unusual case most likely created behavior that was difficult to understand for the perceiving subject. The subject gave a response of 5, suggesting a slight leaning towards gray being dominant. In fact, gray was slightly more dominant, feeling 0.12 dominance towards white, while white felt 0.08 dominant towards gray. However, gray had a higher confidence in its submissive state ( 0.87 vs. white's 0.65 ), so the weighted average showed a slight lean towards gray being more submissive $(0.46)$.

\section{References}

Axelrod, R. (1984) The Evolution of

Cooperation, Basic Books, New York, USA.

Bates, J., Loyall, A. and Reilly, W. (1992) An

Architecture for Action, Emotion, and
Social Behavior, Lecture Notes in Computer Science Vol. 830: Selected Papers from the Fourth European Workshop on Modeling Autonomous Agents in a Multi-Agent World, pp. 55-68.

Beaver, B. V. (1999) Canine Behavior: A Guide for Veterinarians, W. B. Saunders Company, Philadelphia, PA, USA.

Bickmore, T. (2003) Relational Agents: Effecting Change through Human-Computer Relationships, Ph.D. dissertation, Media Arts \& Sciences, Massachusetts Institute of Technology, Cambridge, MA, USA.

Bickmore, T. W. and Picard, R. W. (2004) Towards caring machines, In Extended abstracts of the 2004 conference on Human factors and computing systems, ACM Press, pp. 1489-1492.

Blumberg, B., Downie, M., Ivanov, Y., Berlin, M., Johnson, M. P. and Tomlinson, B. (2002) Integrated learning for interactive synthetic characters, Proceedings of the 29th annual conference on Computer graphics and interactive techniques, San Antonio, Texas, USA, July 23-26, 2002, pp. 417-426. boyd, d. m. (2004) Friendster and publicly articulated social networking, In Extended abstracts of the 2004 conference on Human factors and computing systems, ACM Press, pp. 1279-1282.

Breazeal, C. (2000) Sociable Machines: Expressive Social Exchange Between Robot and Human, Sc.D. Dissertation, Artificial Intelligence Laboratory, MIT, Cambridge, MA, USA.

Breazeal, C., Buchsbaum, D., Gray, J., Gatenby, D. and Blumberg, B. (2004) Learning from and about Others: Towards Using Imitation to Bootstrap the Social Understanding of Others by Robots, In: L. Rocha and F. Almedia e Costa (eds.) Artificial Life (to appear).

Burke, R., Isla, D., Downie, M., Ivanov, Y. and Blumberg, B. (2001) CreatureSmarts: The Art and Architecture of a Virtual Brain, Proceedings of the Game Developers Conference, San Jose, CA, USA, March 2024, pp. 147-166. 
Cassell, J., Bickmore, T., Billinghurst, M., Campbell, L., Chang, K., Vilhjálmsson, H. and Yan, H. (1999) Embodiment in Conversational Interfaces: Rea, Proceedings of the CHI'99 Conference on Human Factors in Computing Systems, Pittsburgh, PA, USA, May 15-20, pp. 520-527.

Cavazza, M., Charles, F. and Mead, S. J. (2002) Interacting with Virtual Characters in interactive storytelling, Proceedings of the first international joint conference on autonomous agents and multiagent systems, Bologna, Italy, July 15-19, pp. 318-325.

Cohen, M. D., Riolo, R. L. and Axelrod, R. (1999) The Emergence of Social Organization in the Prisoner's Dilemma: How Context-Preservation and Other Factors Promote Cooperation, Santa Fe Institute Working Paper 99-01-002 (technical report). Available at: http://www.santafe.edu/research/publication s/wpabstract/199901002 (January, 2005).

Damasio, A. (1994) Descartes' Error: Emotion, Reason, and the Human Brain, G. P. Putnam's Sons, New York, NY, USA.

Darwin, C. (1965 (originally published 1872)) The Expression of the Emotions in Man and Animals, The University of Chicago Press, Chicago, IL, USA.

Dautenhahn, K. (2000) Socially Intelligent Agents and The Primate Social Brain Towards a Science of Social Minds., 2000 AAAI Fall Symposium: Socially Intelligent Agents: The Human in the Loop, North Falmouth, MA, USA, November 3-5, pp. 35-51.

Downie, M. (2001) behavior, animation, music: the music and movement of synthetic characters, S.M. Thesis, Media Arts \& Sciences, MIT, Cambridge, MA, USA.

Ekman, P. (1992) An Argument for Basic Emotions, In Basic Emotions, (Ed, Stein, N., and Oatley, K.), Lawrence Erlbaum, Hove, UK, pp. 169-200.

Eladhari, M. and Lindley, C. (2003) Player Character Design Facilitating Emotional Depth in MMORPGs, Digital Games Research Conference 2003, University of
Utrecht, The Netherlands, 4-6 November 2003, pp.

Fisher, D. and Dourish, P. (2004) Social and temporal structures in everyday collaboration, Proceedings of the CHI2004 conference on human factors in computing systems, Vienna, Austria, April 24-29, pp. 551-558.

Fox, M. W. (1971) Behaviour of Wolves, Dogs and Related Canids, Harper \& Row, New York, NY, USA.

Funge, J., Tu, X. and Terzopoulos, D. (1999) Cognitive modeling: knowledge, reasoning and planning for intelligent characters, Proceedings of the 26th annual conference on Computer graphics and interactive techniques (SIGGRAPH 99), Los Angeles, CA, USA, August 8-13, pp. 29-38.

Gamasutra.com (2004a) Latest US Console Games Sales Charts, 7/26/04, Available at: http://www.gamasutra.com/phpbin/news_index.php?story=4070 (January, 2005).

Gamasutra.com (2004b) Latest US PC Games Sales Charts, 7/28/04, Available at: http://www.gamasutra.com/phpbin/news_index.php?story=4081 (January, 2005).

Gratch, J., Rickel, J., André, E., Badler, N., Cassell, J. and Petajan, E. (2002) Creating Interactive Virtual Humans: Some Assembly Required, IEEE Intelligent Systems, July/August, pp. 54-63.

Hales, D. and Edmonds, B. (2003) Evolving social rationality for MAS using "tags", Proceedings of the second international joint conference on Autonomous agents and multiagent systems, Melbourne, Australia, July 14-18, pp. 497-503.

Hemelrijk, C. K. (1996) Dominance interactions, spatial dynamics and emergent reciprocity in a virtual world., From Animals to Animats 4: Proceedings of the Fourth International Conference on Simulation of Adaptive Behavior, Cape Cod, MA, USA, September 9-13, pp. 545-552.

Hodgins, J. K. and Pollard, N. S. (1997) Adapting simulated behaviors for new characters, Proceedings of the 24th annual 
conference on Computer graphics and interactive techniques (SIGGRAPH 97), Los Angeles, CA, USA, August 3-8, pp. 153162.

Immerman, R. S. and Mackey, W. C. (2003) Perspectives on human attachment (pair bonding): Eve's unique legacy of a canine analogue, Evolutionary Psychology, 1 pp. 138-154.

Isla, D., Burke, R., Downie, M. and Blumberg, B. (2001) A Layered Brain Architecture for Synthetic Creatures, Proceedings of the International Joint Conferences on Artificial Intelligence (IJCAI), Seattle, WA, USA, August 4-10, pp. 1051-1058.

Jenkins, H. (2002) Game Theory: Digital Renaissance, MIT Technology Review, pp. 1-3.

Klinghammer, E. and Goodmann, P. (1985) The Management and Socialization of Captive Wolves (Canis lupus) at Wolf Park, North American Wildlife Park Foundation, Inc., Battle Ground, IN, USA.

Kollock, P. (1998) Design Principles for Online Communities, PC Update, 15, (5), pp. 5860.

Lee, J., Chai, J., Reitsma, P. S. A., Hodgins, J. K. and Pollard, N. S. (2002) Interactive control of avatars animated with human motion data, In SIGGRAPH '02:

Proceedings of the 29th annual conference on Computer graphics and interactive techniques, ACM Press, pp. 491-500.

Mateas, M. and Stern, A. (2003) Towards Building a Fully Realized Interactive Drama, Game Developers Conference, San Jose, CA, USA, March 6-8, pp. 24.

Mech, L. D. (1999) Alpha status, dominance, and division of labor in wolf packs., Canadian Journal of Zoology, 77 pp. 11961203.

Mech, L. D., Adams, L. G., Meier, T. J., Burch, J. W. and Dale, B. W. (1998) The Wolves of Denali, University of Minnesota Press, Minneapolis, MN, USA.

Mehrabian, A. and Russell, J. (1974) An Approach to Environmental Psychology, MIT Press, Cambridge, MA, USA.
Nardi, B. (2005) Beyond Bandwidth: Dimensions of Connection in Interpersonal Communication, Journal of ComputerSupported Cooperative Work, (in press).

Paiva, A., Machado, I. and Prada, R. (2001) Heroes, villians, magicians...: dramatis personae in a virtual story creation environment, Proceedings of the 6th international conference on Intelligent user interfaces, Santa Fe, NM, USA, January 1417, pp. 129-136.

Panksepp, J. (1998) Affective Neuroscience: The Foundations of Human and Animal Emotions, Oxford University Press, New York, NY, USA.

Perlin, K. and Goldberg, A. (1996) Improv: A System for Scripting Interactive Actors in Virtual Worlds, Proceedings of ACM SIGGRAPH 96, New Orleans, LA, USA, August 4-9, pp. 205-216.

Prendinger, H., Mori, J., Saeyor, S., Mori, K., Okazaki, N., Juli, Y., Mayer, S., Dohi, H. and Ishizuka, M. (2004) Scripting and Evaluating Affective Interactions with Embodied Conversational Agents, Künstliche Intelligenz (KI) Zeitschrift, 1 pp. 4-10.

Randall, N. and Pedersen, I. (1998) Who exactly is trying to help us? The ethos of help systems in popular computer applications, Proceedings of the 16th annual international conference on Computer documentation, Quebec, Quebec, CA, September 24 - 26, pp. 63-69.

Raybourn, E. M. (2004). Designing Intercultural Agents for Multicultural Interactions. In Sabine Payr \& Robert Trappl (Eds.), Agent Culture: Human-Agent Interaction in a Multicultural World, Lawrence Erlbaum \& Associates, Mahwah, NJ, USA, pp. 267-285.

Reeves, B. and Nass, C. (1996) The Media Equation, Cambridge University Press, Cambridge, UK.

Reilly, W. S. N. (1996) Believable Social and Emotional Agents, Ph.D. dissertation, School of Computer Science, Carnegie Mellon University, Pittsburgh, PA, USA. Reynolds, C. (1987) Flocks, Herds and Schools: A Distributed Behavioral Model, 
Proceedings of ACM SIGGRAPH 87, Anaheim, CA, USA, July 27-31, pp. 25-34. Schaub, H., Zoll, C. and Aylett, R. (2003) Modelling Empathy: The EU-project VICTEC (Virtual Information and Communication Technology with Empathic Characters), Fifth International Conference on Cognitive Modeling, Bamberg, Germany, April 10-12, pp. 291-294.

Shannon, C. E. (1948) A Mathematical Theory of Communication, The Bell System Technical Journal, 27, (July, October, 1948), pp. 379-423, 623-656.

Thalmann, D., Noser, H. and Huang, Z. (1997) Autonomous Virtual Actors Based on Virtual Sensors, Creating Personalities, Heidelberg, Germany, pp. 25-42.

Tomlinson, B. and Blumberg, B. (2002) Social synthetic characters (Visfiles column, edited by Bill Hibbard), SIGGRAPH Computer Graphics, 36, (2), pp. 5-7.

Tomlinson, B., Blumberg, B. and Nain, D. (2000) Expressive Autonomous Cinematography for Interactive Virtual Environments, Proceedings of 4th Int'l. Conf. on Autonomous Agents, Barcelona, Catalonia, Spain, pp. 317-324.

Tomlinson, B., Downie, M., Berlin, M., Gray, J., Wong, A., Burke, R., Isla, D., Ivanov, Y., Johnson, M. P., Lyons, D., Cochran, J., Yong, B., Stiehl, D., Soetjipto, R., Zaharopol, D. and Blumberg, B. (2001) AlphaWolf, Proceedings of SIGGRAPH 2001: conference abstracts and applications, pp. 2.

Tomlinson, W. (2002) Synthetic Social Relationships for Computational Entities, Ph.D. dissertation, Media Arts \& Sciences, Massachusetts Institute of Technology, Cambridge, MA, USA.

US_Army (2004) The United States Army, Available at: http://www.army.mil/ (December 2004).

Vaughan, R. T., Stoy, K., Sukhatme, G. S. and Mataric, M. J. (2000) Go ahead, make my day: robot conflict resolution by aggressive competition, In From Animals to Animats 6: Proc. Int. Conf. Simulation of Adaptive Behaviour, (Ed, Berthoz A, F. D., Meyer J,
Roitblat H, Wilson S), MIT Press, Cambridge, MA, USA, pp. 491-500.

Velasquez, J. (1998) When Robots Weep: Emotional Memories and Decision-Making, Proceedings of the Fifteenth National Conference on Artificial Intelligence, Madison, WI, USA, pp. 70-75.

Watts, D. J. (2003) Six Degrees: The Science of a Connected Age, W.W. Norton \& Company, New York, NY, USA. Wrangham, R. W., McGrew, W. C., de Waal, F. B. M. and Heltne, P. G. (Eds.) (1994) Chimpanzee Cultures, Harvard University Press, Cambridge, MA, USA. 